Louisiana State University

LSU Digital Commons

Faculty Publications

Department of Mathematics

$1-18-2019$

\title{
Sequential Predictors for Linear Time-Varying Systems with Delays in the Vector Field and in the Input
}

Michael Malisoff

Louisiana State University

Frederic Mazenc

Laboratoire des Signaux et Systèmes

Follow this and additional works at: https://digitalcommons.Isu.edu/mathematics_pubs

\section{Recommended Citation}

Malisoff, M., \& Mazenc, F. (2019). Sequential Predictors for Linear Time-Varying Systems with Delays in the Vector Field and in the Input. Proceedings of the IEEE Conference on Decision and Control, 2018-December, 6246-6249. https://doi.org/10.1109/CDC.2018.8619238

This Conference Proceeding is brought to you for free and open access by the Department of Mathematics at LSU Digital Commons. It has been accepted for inclusion in Faculty Publications by an authorized administrator of LSU Digital Commons. For more information, please contact ir@lsu.edu. 


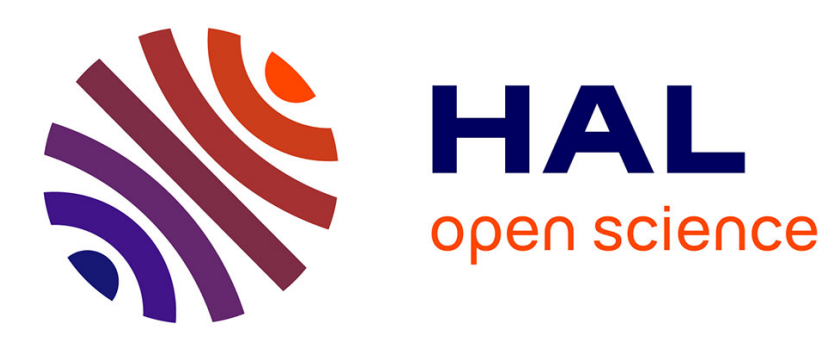

\title{
Sequential Predictors for Linear Time-Varying Systems with Delays in the Vector Field and in the Input
}

\author{
Michael Malisoff, Frédéric Mazenc
}

\section{To cite this version:}

Michael Malisoff, Frédéric Mazenc. Sequential Predictors for Linear Time-Varying Systems with Delays in the Vector Field and in the Input. 57th IEEE Conference on Decision and Control (CDC 2018), Dec 2018, Miami, United States. hal-01918343

\section{HAL Id: hal-01918343 \\ https://hal.inria.fr/hal-01918343}

Submitted on 10 Nov 2018

HAL is a multi-disciplinary open access archive for the deposit and dissemination of scientific research documents, whether they are published or not. The documents may come from teaching and research institutions in France or abroad, or from public or private research centers.
L'archive ouverte pluridisciplinaire HAL, est destinée au dépôt et à la diffusion de documents scientifiques de niveau recherche, publiés ou non, émanant des établissements d'enseignement et de recherche français ou étrangers, des laboratoires publics ou privés. 


\section{Sequential Predictors for Linear Time-Varying Systems with Delays in the Vector Field and in the Input}

\author{
Michael Malisoff
}

\author{
Frederic Mazenc
}

\begin{abstract}
We provide new sequential predictors for a large class of linear time-varying systems that contain constant delays in the vector fields and also constant delays in the inputs. We allow the input delays to be arbitrarily large. We prove global exponential stability of the origin for an augmented system that includes the original system in closed loop with our sequential predictors based feedback control. We illustrate our new theorem in an example from identification theory.
\end{abstract}

Index Terms-Delays, stability, time-varying systems

\section{INTRODUCTION}

This work continues our search for sequential predictors that can help solve feedback stabilization problems that have arbitrarily long input delays, using dynamical extensions that consist of stacks of ordinary differential equations but which do not contain distributed terms. Whereas [13] and [12] were confined to nonlinear systems with constant delays, and timevarying linear systems with time-varying delays, respectively, and while [23] also covered time-varying systems with sampling and measurement delays, here we study a complementary problem, namely, global exponential feedback stabilization under arbitrarily long input delays in systems that also contain other delays in the vector fields. This problem was addressed in [26], using distributed delays.

Our work is motivated by engineering systems where several delays are present [15]. The work [15] used emulation and Lyapunov-Krasovskii functionals, and imposed upper bounds on the allowable input delays. These bounds are not required in our sequential predictors approach.

Instead of the types of distributed terms that are commonly found in the delay compensation literature (where the feedback control is typically represented as an implicit solution of an integral equation, instead of in a more user friendly closed form), the dynamic extensions in sequential prediction contain time-rescaled copies of the original system (called subpredictors), with each subpredictor also containing added stabilizing terms. The sequential predictor paradigm [5] is a recent development in a long history of works on stabilization under long feedback delays. Pioneering earlier works include the Smith predictor for linear systems [22], and work by Artstein [3], Krstic [11], and other notable researchers through the 1980's and 1990s. See also the more recent works [10], [18], [19], and [24] on delay systems.

\footnotetext{
Malisoff is with the Department of Mathematics, Louisiana State University, Baton Rouge, LA 70803-4918, USA, malisoff@1su.edu.

Mazenc is with EPI DISCO INRIA-Saclay, Laboratoire des Signaux et Systèmes (L2S, UMR CNRS 8506), CNRS, CentraleSupélec, Université Paris-Sud, 3 rue Joliot Curie, 91192, Gif-sur-Yvette, France, frederic.mazenc@12s.centralesupelec.fr.

Corresponding Author: F. Mazenc. Supported by NSF Grant 1711299.
}

Feedback stabilization can be challenging from the theory side, and also has many engineering motivations. See, e.g., the work [16], and [4], [17], and [21] which provide useful surveys of delay compensating control.

The usual prediction approach eliminates feedback delays, by replacing the delayed states in the feedback by predicted values. While standard prediction generally yields controls with distributed terms, see, e.g., [1], [6], [7], and [25] for controllers for special cases of time invariant systems that do not produce distributed terms and that are based on prediction, and the works [8] and [14] on chain observers that do not allow different delays in the vector fields and in the input, and so do not address the problems that we help solve here. To address cases with different delays in the input and the vector fields, this work provides a new class of sequential predictors, consisting of two interconnected subsets of subpredictors. The first subset of $p$ subpredictors compensates for the delay in the vector field. Then a second set of $p(k-1)$ predictors compensates for the input delays, where we assume that the input delay is an integer multiple $k \tau$ of the delay $\tau$ in the vector field for some integer $k \geq 2$.

\section{MAIN RESULT}

We study systems of the form

$$
\dot{x}(t)=A(t) x(t)+B(t) u(t-k \tau)+C(t) x(t-\tau)
$$

where $x$ is valued in $\mathbb{R}^{n}$ for any dimension $n, \tau \geq 0$ is a constant input delay, $k \geq 2$ is a positive integer, and the matrix valued functions $A, B$, and $C$ are assumed to be continuous and bounded. The feedback control $u$ will be specified later, and we write it as a function of $t$ to keep the notation simple. We assume that the initial functions are constant at the initial time, and that the initial time is always zero. Time-varying linear systems of the form (1) arise when linearizing a nonlinear system around a reference trajectory, and can represent a closed loop system with a relatively short delay $\tau>0$ and a longer delay $k \tau$ with the integer $k$ as large as desired. The following assumption agrees with the assumptions from [12] and [13] in the special case of timevarying linear systems with constant delays when $C=0$ :

Assumption 1: There is a bounded continuous matrix valued function $K$ such that the origin of

$$
\dot{x}(t)=A(t) x(t)+B(t) u_{s}(t, x(t))+C(t) x(t-\tau)
$$

with $u_{s}(t, x)=K(t) x$ is uniformly globally exponentially stable.

In many cases, Assumption 1 can be satisfied in practice. For instance, in some cases, one can treat the term 
$\delta(t)=C(t) x(t-\tau)$ as a disturbance and use high gains to dominate this term. Then no condition on the size of $\tau$ is needed. In other situations, one can often determine $K(t)$ so that $\dot{x}(t)=(A(t)+C(t)+B(t) K(t)) x(t)$ is uniformly globally exponentially stable to 0 and prove that $\dot{x}(t)=(A(t)+B(t) K(t)) x(t)+C(t) x(t-\tau)$ is uniformly globally exponentially stable to 0 , when $\tau$ is smaller than a constant $\bar{\tau}$, where $\bar{\tau}$ can be found by an approach that is similar to [9] and [15]. See also Section IV for an example.

On the other hand, since we allow $k$ in (1) to be as large as desired, emulation would not cover our feedback problem for (1), so we use sequential predictor controls. Our main result is as follows, where $I_{n}$ is the identity matrix and $|\cdot|_{\infty}$ is the essential supremum:

Theorem 1: Let Assumption 1 hold, let $\ell>0$ be a constant, and choose any integer

$$
\begin{aligned}
& p> \\
& \max \left\{2, \frac{2 \sqrt{2} \tau}{\ell}\left|A+\ell I_{n}\right|_{\infty} \max \left\{|A|_{\infty},\left|A+\ell I_{n}\right|_{\infty}\right\}\right\} .
\end{aligned}
$$

Set $L(t)=-\left[A(t)+\ell I_{n}\right]$ and $\phi_{i}(t)=t+\frac{i \tau}{p}$ for all $i \in$ $\{1,2, \ldots, p k\}$. Then (1) in closed loop with the control

$$
u_{\text {new }}(t)=K(t+k \tau) z_{p k}(t)
$$

where $z_{p k}$ is the last $n$ components of the $n p k$-dimensional system

$$
\left\{\begin{aligned}
\dot{z}_{i}(t)= & A\left(\phi_{i}(t)\right) z_{i}(t) \\
& +B\left(\phi_{i}(t)\right) u_{\text {new }}\left(\phi_{i}(t)-k \tau\right) \\
& +L\left(\phi_{i}(t)\right)\left[z_{i}\left(t-\frac{\tau}{p}\right)-z_{i-1}(t)\right] \\
& +C\left(\phi_{i}(t)\right) x\left(t-\tau+\frac{i \tau}{p}\right), 1 \leq i \leq p \\
\dot{z}_{p+j}(t)= & A\left(\phi_{p+j}(t)\right) z_{p+j}(t) \\
& +B\left(\phi_{p+j}(t)\right) u_{\text {new }}\left(\phi_{p+j}(t)-k \tau\right) \\
& +L\left(\phi_{p+j}(t)\right)\left[z_{p+j}\left(t-\frac{\tau}{p}\right)-z_{p+j-1}(t)\right] \\
& +C\left(\phi_{p+j}(t)\right) z_{p}\left(t-\tau+\frac{j \tau}{p}\right) \\
& 1 \leq j \leq p(k-1)
\end{aligned}\right.
$$

and $z_{0}=x$, is such that the dynamics for $\left(x(t), z_{1}(t)-x(t+\right.$ $\left.\tau / p), z_{2}(t)-z_{1}(t+\tau / p), \ldots, z_{p k}(t)-z_{p k-1}(t+\tau / p)\right)$ is uniformly globally exponentially stable to 0 .

Remark 1: The $z_{i}$ dynamics in (5) is called the $i$ th sequential predictor for each $i$. Formula (3) implies that when $A$ is the zero function, we can choose any $p \geq 2$, by picking $\ell>0$ small enough. However, Theorem 1 also applies under nonzero $A$ 's, by choosing a large enough $p$ such that (3) holds (since the right side of (3) does not depend on $p$ ).

\section{Proof of Main REsult}

The proof has three steps. First, we prove that the system

$$
\dot{\xi}(t)=A\left(\phi_{i}(t)\right) \xi(t)+L\left(\phi_{i}(t)\right) \xi\left(t-\frac{\tau}{p}\right)
$$

is uniformly globally exponentially stable to 0 for each fixed choice of $i$, when (3) is satisfied. In the second step, we show that the dynamics for $\left(\bar{z}_{1}, \ldots, \bar{z}_{p}\right)$ with the choices

$$
\bar{z}_{i}(t)=z_{i}(t)-z_{i-1}\left(t+\frac{\tau}{p}\right)
$$

for $i=1,2, \ldots, p$ is uniformly globally exponentially stable to 0 . Combining the first two steps allows us to prove the conclusion of the theorem in our third step. We use the fact that for each constant $c_{*}>0$ and each continuous function $q:\left[-c_{*}, \infty\right) \rightarrow[0, \infty)$, the conditions

$$
\begin{array}{r}
\frac{d}{d t} \int_{t-c_{*}}^{t} \int_{\ell}^{t} q(r) \mathrm{d} r \mathrm{~d} \ell=c_{*} q(t)-\int_{t-c_{*}}^{t} q(r) \mathrm{d} r \\
\quad \text { and } \int_{t-c_{*}}^{t} \int_{\ell}^{t} q(r) \mathrm{d} r \mathrm{~d} \ell \leq c_{*} \int_{t-c_{*}}^{t} q(r) \mathrm{d} r
\end{array}
$$

hold for all $t \geq 0$.

First Step. We first show that (3) implies that (6) is uniformly globally exponentially stable to 0 , using the following variant of a Lyapunov-Krasovskii functional argument from [15]. Our strategy is to first rewrite (6) in the form

$$
\dot{\xi}(t)=-\ell \xi(t)-\left[A\left(\phi_{i}(t)\right)+\ell I_{n}\right]\left(\xi\left(t-\frac{\tau}{p}\right)-\xi(t)\right)
$$

and to note that the time derivatives of $V(\xi)=\frac{1}{2}|\xi|^{2}$ and

$V^{\sharp}\left(\xi_{t}\right)=V(\xi(t))$
$+\frac{2 \tau}{\ell p}|L|_{\infty}^{2}\left(\max \left\{|A|_{\infty},|L|_{\infty}\right\}\right)^{2} \int_{t-2 \tau / p}^{t} \int_{s}^{t}|\xi(\ell)|^{2} \mathrm{~d} \ell \mathrm{d} s$

along all solutions of (9) for all $t \geq 0$ satisfy

$$
\begin{aligned}
\frac{d}{d t} V(\xi(t))= & -\ell|\xi(t)|^{2}-\xi(t)\left[A\left(\phi_{i}(t)\right)+\ell I_{n}\right] \\
& \times\left(\xi\left(t-\frac{\tau}{p}\right)-\xi(t)\right) \\
\leq & -\ell|\xi(t)|^{2} \\
& +|\xi(t)|\left|A+\ell I_{n}\right|_{\infty} \int_{t-\tau / p}^{t}|\dot{\xi}(\ell)| \mathrm{d} \ell \\
\leq & -\ell|\xi(t)|^{2}+|\xi(t)|\left|A+\ell I_{n}\right|_{\infty} \\
& \times \max \left\{|A|_{\infty},|L|_{\infty}\right\} \int_{t-2 \tau / p}^{t}|\xi(\ell)| \mathrm{d} \ell \\
\leq & -\frac{\ell}{2}|\xi(t)|^{2}+\frac{1}{2 \ell}\left|A+\ell I_{n}\right|_{\infty}^{2} \\
& \times\left(\max \left\{|A|_{\infty},|L|_{\infty}\right\}\right)^{2} \frac{2 \tau}{p} \\
& \times \int_{t-2 \tau / p}^{t}|\xi(\ell)|^{2} \mathrm{~d} \ell,
\end{aligned}
$$

by Hölder's inequality $a b \leq \frac{\ell}{2} a^{2}+\frac{1}{2 \ell} b^{2}$ with $a=|\xi(t)|$, followed by Jensen's inequality, and therefore also

$$
\begin{aligned}
& \frac{d}{d t} V^{\sharp}\left(\xi_{t}\right) \\
& \leq-\left[\frac{\ell}{2}-\frac{4 \tau^{2}}{p^{2} \ell}\left|A+\ell I_{n}\right|_{\infty}^{2} \max \left\{|A|_{\infty}^{2},|L|_{\infty}^{2}\right\}\right]|\xi(t)|^{2} \\
& -\frac{\tau}{p \ell}\left|A+\ell I_{n}\right|_{\infty}^{2} \max \left\{|A|_{\infty}^{2},|L|_{\infty}^{2}\right\} \int_{t-2 \tau / p}^{t}|\xi(\ell)|^{2} \mathrm{~d} \ell
\end{aligned}
$$

where $\xi_{t}$ is defined by $\xi_{t}(s)=\xi(t+s)$ for all values $t \geq 0$ and $s \leq 0$, by applying (8a) with the choices $c_{*}=2 \tau / p$ and $q(r)=|\xi(r)|^{2}$. Since (3) ensures that the quantity in squared brackets in (12) is positive, we can then use (8b) with the same choices of $c_{*}$ and $q$ to find a positive constant $c_{a}$ such that $\frac{d}{d s} V^{\sharp}\left(\xi_{s}\right) \leq-c_{a} V^{\sharp}\left(\xi_{s}\right)$ along all solutions of (9) for all $s \geq 0$, which we can then integrate on any interval $[0, t]$ to get the desired uniform global exponential stability condition on (6), using the quadratic structure of $V$ and our assumption that the initial functions are constant.

Second Step. We prove that the dynamics for $\bar{z}_{a}=$ $\left(\bar{z}_{1}, \ldots, \bar{z}_{p}\right)$ defined in terms of the error components (7) is uniformly globally exponentially stable to zero, which will follow from the first step of the proof and our choices of the arguments $\phi_{i}(t)$ in the time-varying coefficients $A, B, C$, and $L$ in our sequential predictor dynamics (5). First note 
that the closed loop $x$ subsystem in Theorem 1 satisfies

$$
\begin{aligned}
& \frac{d}{d t} x\left(\phi_{1}(t)\right)=A\left(\phi_{1}(t)\right) x\left(\phi_{1}(t)\right) \\
& +B\left(\phi_{1}(t)\right) u_{\text {new }}\left(\phi_{1}(t)-k \tau\right)+C\left(\phi_{1}(t)\right) x\left(\phi_{1}(t)-\tau\right)
\end{aligned}
$$

which produces the subsystem $\dot{\bar{z}}_{1}(t)=A\left(\phi_{1}(t)\right) \bar{z}_{1}(t)+$ $L\left(\phi_{1}(t)\right) \bar{z}_{1}(t-(\tau / p))$, which is uniformly globally exponentially stable to 0 , by the first step of the proof. Reasoning inductively then shows that the dynamics

$$
\left\{\begin{aligned}
\dot{\bar{z}}_{1}(t)= & A\left(\phi_{1}(t)\right) \bar{z}_{1}(t)+L\left(\phi_{1}(t) \bar{z}_{1}\left(t-\frac{\tau}{p}\right)\right. \\
\dot{\bar{z}}_{i}(t)= & A\left(\phi_{i}(t)\right) \bar{z}_{i}(t)+L\left(\phi_{i}(t)\right) \bar{z}_{i}\left(t-\frac{\tau}{p}\right) \\
& -L\left(\phi_{i}(t)\right) \bar{z}_{i-1}(t), i=2, \ldots, p
\end{aligned}\right.
$$

for $\bar{z}_{a}$ are uniformly globally exponentially stable to 0 , by noting that each of the systems

$$
\dot{\bar{z}}_{i}(t)=A\left(\phi_{i}(t)\right) \bar{z}_{i}(t)+L\left(\phi_{i}(t)\right) \bar{z}_{i}\left(t-\frac{\tau}{p}\right)+\delta(t)
$$

for $i=2, \ldots, p$ is exponentially input-to-state stable with respect to the disturbance $\delta$, since $V^{\sharp}$ from the first step of the proof is also an input-to-state stability Lyapunov-Krasovskii functional for (14) and $L$ is a bounded function.

Third Step. We prove the theorem using the second step of the proof, by setting $\zeta(t)=z_{p}(t-\tau)-x(t)$ and first rewriting the $\left(z_{p+1}, \ldots z_{p k}\right)$ subsystem of (5) as

$$
\begin{aligned}
\dot{z}_{p+\ell}(t)= & A\left(\phi_{p+\ell}(t)\right) z_{p+\ell}(t) \\
& +B\left(\phi_{p+\ell}(t)\right) u_{\text {new }}\left(\phi_{p+\ell}(t)-k \tau\right) \\
& +L\left(\phi_{p+\ell}(t)\right)\left[z_{p+\ell}\left(t-\frac{\tau}{p}\right)-z_{p+\ell-1}(t)\right] \\
& +C\left(\phi_{p+\ell}(t)\right)\left[x\left(t+\frac{\ell \tau}{p}\right)\right. \\
& \left.+\zeta\left(t+\frac{\ell \tau}{p}\right)\right], 1 \leq \ell \leq p(k-1) .
\end{aligned}
$$

We also use the fact that for all $t \geq 0$, we have

$$
\begin{aligned}
& \bar{z}_{p}\left(t-\tau+\frac{\tau}{p}\right)+\bar{z}_{p-1}\left(t-\tau+\frac{2 \tau}{p}\right)+\ldots+\bar{z}_{1}(t) \\
& =z_{p}\left(t-\tau+\frac{\tau}{p}\right)-x\left(t+\frac{\tau}{p}\right)=\zeta\left(t+\frac{\tau}{p}\right)
\end{aligned}
$$

which follows from the pairwise cancellation of terms in the sum of the $p$ terms in a telescoping sum. Using (15), we can rewrite the dynamics for $\bar{z}_{b}=\left(\bar{z}_{p+1}, \ldots, \bar{z}_{p k}\right)$ as

$$
\begin{aligned}
\dot{\bar{z}}_{p+1}(t)= & A\left(\phi_{p+1}(t)\right) \bar{z}_{p+1}(t) \\
& +L\left(\phi_{p+1}(t)\right)\left(\bar{z}_{p+1}\left(t-\frac{\tau}{p}\right)-\bar{z}_{p}(t)\right) \\
& +C\left(\phi_{p+1}(t)\right) \zeta\left(t+\frac{\tau}{p}\right) \\
\dot{\bar{z}}_{p+\ell}(t)= & A\left(\phi_{p+\ell}(t)\right) \bar{z}_{p+\ell}(t) \\
& +L\left(\phi_{p+\ell}(t)\right) \bar{z}_{p+\ell}\left(t-\frac{\tau}{p}\right) \\
& -L\left(\phi_{p+\ell}(t)\right) \bar{z}_{p+\ell-1}(t), \\
& 2 \leq \ell \leq p(k-1),
\end{aligned}
$$

since our formulas for the last $p(k-1)$ sequential predictors in (5) ensure that there are no $C$ terms in the dynamics for $\bar{z}_{p+2}, \ldots, \bar{z}_{p k}$. Using the uniform global exponential stability of the dynamics (13) for $\bar{z}_{a}$, the telescoping sum (16), and the exponential input-to-state stability of (14) with respect to $\delta$, it follows that the dynamics for $\left(\bar{z}_{a}, \bar{z}_{b}\right)=\left(\bar{z}_{1}, \ldots, \bar{z}_{p k}\right)$ is uniformly globally exponentially stable to 0 .

Moreover, we can write $z_{p k}(t)=x(t+k \tau)+\sigma(t)+\zeta(t+$ $k \tau)$ in terms of the sum

$$
\sigma(t)=\sum_{s=0}^{p(k-1)-1} \bar{z}_{p k-s}\left(t+\frac{s \tau}{p}\right)
$$

for all $t \geq 0$ (using a telescoping sum argument as we did to obtain (16)). Then the dynamics for the combined variable $\left(x(t), \bar{z}_{1}(t), \ldots, \bar{z}_{p k}(t)\right)=\left(x(t), z_{1}(t)-x(t+\tau / p), z_{2}(t)-\right.$ $\left.z_{1}(t+\tau / p), \ldots, z_{p k}(t)-z_{p k-1}(t+\tau / p)\right)$ are

$$
\left\{\begin{aligned}
\dot{x}(t)= & A(t) x(t)+C(t) x(t-\tau) \\
& +B(t) u_{s}(t, x(t)+\sigma(t-k \tau)+\zeta(t))
\end{aligned}\right.
$$

interconnected with (13) and (17). Since (16) and (18) express $\sigma$ and $\zeta$ as linear combinations of the components of $\left(\bar{z}_{a}, \bar{z}_{b}\right)$, and the $\left(\bar{z}_{a}, \bar{z}_{b}\right)$ subsystem of (19) is uniformly globally exponentially stable to 0 , it follows from Assumption 1 and the linearity of $u_{s}$ in $x$ that (19) is uniformly globally exponentially stable to 0 , which proves the theorem.

\section{ILLUSTRATION}

Consider the dynamics from identification theory (from [2], [15], [20] and several references contained therein)

$$
\dot{x}=-m(t) m^{\top}(t) u
$$

with a control $u$ where $x$ is valued in $\mathbb{R}^{n}$ for any dimension $n$, under the following assumption from [15]:

Assumption 2: The function $m: \mathbb{R} \rightarrow \mathbb{R}^{n}$ is continuous, there are constants $\alpha^{\prime} \in(0,1), \beta^{\prime}>0$, and $\tilde{c}>0$ such that

$$
\alpha^{\prime} I_{n} \leq \int_{t}^{t+\tilde{c}} m(\tau) m^{\top}(\tau) \mathrm{d} \tau \leq \beta^{\prime} I_{n} \text { for all } t \in \mathbb{R},
$$

and $|m(t)|=1$ for all $t \in \mathbb{R}$.

We can build a strict Lyapunov function $V$ for (20) in closed loop with $u_{s}(t, x)=x$, using this lemma from [15]:

Lemma 1: Let $m, \alpha^{\prime}, \beta^{\prime}$, and $\tilde{c}$ be such that Assumption 2 is satisfied. Then, with the choices $\kappa=1+\frac{\tilde{c}}{2}+\frac{1}{4 \alpha^{\prime}} \tilde{c}^{4}$ and

$$
P(t)=\kappa I_{n}+\int_{t-\tilde{c}}^{t} \int_{s}^{t} m(l) m^{\top}(l) \mathrm{d} l \mathrm{~d} s,
$$

the function $V(t, x)=x^{\top} P(t) x$ satisfies $\dot{V} \leq-\alpha^{\prime}|x|^{2} / 2$ along all trajectories of $\dot{x}(t)=-m(t) m^{\top}(t) x(t)$ for all $t \geq$ 0 . Moreover, $|P|_{\infty} \leq \kappa+\tilde{c}^{2}$.

Under the preceding assumptions, [15] provided a bound $\bar{\tau}$ such that $\dot{x}(t)=-m(t) m^{\top}(t) u_{s}\left(t, x_{1}\left(t-\tau_{1}\right), \ldots, x_{n}(t-\right.$ $\left.\left.\tau_{n}\right)\right)$ is uniformly globally asymptotically stable to 0 for all constant delays $\tau_{i} \in[0, \bar{\tau}]$ for $i=1,2, \ldots, n$. Also, since (20) has no drift term, [15, Remark 4] showed how to allow arbitrarily large $\bar{\tau}$ by scaling the control, but this control scaling approach may not be viable in practice. Therefore, we illustrate Theorem 1 above for the input delayed version

$$
\dot{x}(t)=-M_{a}(t) u(t-k \tau)-M_{b}(t) x_{b}(t-\tau)
$$

where $x=\left(x_{a}^{\top}, x_{b}^{\top}\right)^{\top}$ for any dimensions $n_{a}$ and $n_{b}$ for $x_{a}$ and $x_{b}$ respectively such that $n=n_{a}+n_{b}$, and $M_{a}(t)$ 
(resp., $M_{b}(t)$ ) consists of the first $n_{a}$ (resp., last $n_{b}$ ) columns of $m(t) m^{\top}(t)$ for all $t \in \mathbb{R}$. We use the following lemma:

Lemma 2: Let the assumptions of Lemma 1 hold, and $M_{a}$, $M_{b}, \alpha^{\prime}, \kappa$, and $\tilde{c}$ be defined as above and $\tau>0$ be a constant such that

$$
\tau<\frac{\alpha^{\prime}}{4 \sqrt{5}\left(\kappa+\tilde{c}^{2}\right)}
$$

Then Assumption 1 is satisfied with $A=0_{n \times n}, B=$ $-\left[M_{a} 0_{n \times n_{b}}\right], C=-\left[0_{n \times n_{a}} M_{b}\right]$, and $u_{s}(t, x)=x$.

Proof: By writing

$$
\dot{x}(t)=-M_{a}(t) x_{a}(t)-M_{b}(t) x_{b}(t-\tau)
$$

in the form $\dot{x}(t)=-m(t) m^{\top}(t) x(t)+M_{b}(t)\left[x_{b}(t)-x_{b}(t-\right.$ $\tau)$ ] and using the bound $|M|_{\infty} \leq 1$, it follows that the time derivative of the function $V$ from Lemma 1 along all solutions of (25) for all $t \geq 0$ satisfies

$$
\begin{aligned}
\dot{V} & \leq-\frac{\alpha^{\prime}|x(t)|^{2}}{2}+\{|x(t)|\}\left\{2\left(\kappa+\tilde{c}^{2}\right) \int_{t-2 \tau}^{t}|x(\ell)| \mathrm{d} \ell\right\} \\
& \leq-\frac{\alpha^{\prime}|x(t)|^{2}}{4}+4\left(\kappa+\tilde{c}^{2}\right)^{2} \frac{1}{\alpha^{\prime}}(2 \tau) \int_{t-2 \tau}^{t}|x(\ell)|^{2} \mathrm{~d} \ell,
\end{aligned}
$$

by applying the inequality $c_{1} c_{2} \leq \frac{\alpha^{\prime}}{4} c_{1}^{2}+\frac{1}{\alpha^{\prime}} c_{2}^{2}$ where $c_{1}$ and $c_{2}$ are the terms in curly braces in (26) followed by Jensen's inequality. Therefore, the time derivative of

$$
V^{\sharp}\left(x_{t}\right)=V(x(t))+5\left(\kappa+\tilde{c}^{2}\right)^{2} \frac{2 \tau}{\alpha^{\prime}} \int_{t-2 \tau}^{t} \int_{\ell}^{t}|x(s)|^{2} \mathrm{~d} s \mathrm{~d} \ell
$$

along all solutions of (25) for all $t \geq 0$ satisfies

$$
\begin{aligned}
\frac{d}{d t} V^{\sharp}\left(x_{t}\right) \leq & -\left\{\frac{\alpha^{\prime}}{4}-5\left(\kappa+\tilde{c}^{2}\right)^{2} \frac{4 \tau^{2}}{\alpha^{\prime}}\right\}|x(t)|^{2} \\
& -\left(\kappa+\tilde{c}^{2}\right)^{2} \frac{2 \tau}{\alpha^{\prime}} \int_{t-2 \tau}^{t}|x(\ell)|^{2} \mathrm{~d} \ell,
\end{aligned}
$$

by (8a) with $c_{*}=2 \tau$ and $q(\ell)=|x(\ell)|^{2}$. The constant in curly braces in (27) is positive, by (24). Hence, we can reason as in the last part of the first step in the proof of Theorem 1 to get an exponential decay estimate on $V^{\sharp}$ and so also on $|x(t)|$ all solutions of (23), which proves the lemma.

Since Assumption 1 is satisfied, the uniformly globally exponentially stabilizing feedback control for (23) is given by Theorem 1, for any $k \geq 2$ and any $\tau$ satisfying (24). This allows arbitrarily large delays $k \tau$. Moreover, since Assumption 1 is satisfied with $A=0$, we can choose $p=2$, which produces $2 k$ sequential predictors; see Remark 1.

\section{CONCLUSION}

We provided uniformly globally exponentially stabilizing sequential predictor feedbacks for time-varying linear systems that have delays in the vector field and a longer delay in the feedback. We illustrated how such systems arise when different components of the feedback control can have different delays, which were beyond the scope of the existing sequential predictor based feedback control designs. We plan to extend this work to time-varying delays using analogs of our methods for time-varying delays from [12]. We also plan generalizations with nonlinear systems and measurement delays and sampling. This would extend our sequential predictors works [13] and [23] which did not allow different delays in the vector fields.

\section{REFERENCES}

[1] T. Ahmed-Ali, E. Cherrier, and F. Lamnabhi-Lagarrigue. Cascade high gain predictors for a class of nonlinear systems. IEEE Transactions on Automatic Control, 57(1):221-226, 2012.

[2] B. Anderson. Exponential stability of linear equations arising in adaptive identification. IEEE Transactions on Automatic Control, 22(1):83-88, 1977.

[3] Z. Artstein. Linear systems with delayed controls: a reduction. IEEE Transactions on Automatic Control, 27(4):869-879, 1982.

[4] N. Bekiaris-Liberis and M. Krstic. Nonlinear Control Under Nonconstant Delays. SIAM, Philadelphia, PA, 2013.

[5] G. Besancon, D. Georges, and Z. Benayache. Asymptotic state prediction for continuous-time systems with delayed input and application to control. In Proceedings of the European Control Conference, pages 1786-1791, Kos, Greece, 2007.

[6] F. Cacace, F. Conte, and A. Germani. Memoryless approach to the LQ and LQG problems with variable input delay. IEEE Transactions on Automatic Control, 61(1):216-221, 2016.

[7] F. Cacace, F. Conte, A. Germani, and G. Palombo. Optimal control of linear systems with large and variable input delays. Systems and Control Letters, 89:1-7, 2016.

[8] F. Cacace, A. Germani, and C. Manes. A chain observer for nonlinear systems with multiple time-varying measurement delays. SIAM Journal on Control and Optimization, 52(3):1862-1885, 2014.

[9] E. Fridman, A. Seuret, and J-P. Richard. Robust sampled-data stabilization of linear systems: An input delay approach. Automatica, 40(8):1441-1446, 2004.

[10] H. Ito, Z-P. Jiang, and P. Pepe. Construction of Lyapunov-Krasovskii functionals for networks of iISS retarded systems in small-gain formulation. Automatica, 49(11):3246-3257, 2013.

[11] M. Krstic. Delay Compensation for Nonlinear, Adaptive, and PDE Systems. Birkhauser, Boston, 2009.

[12] F. Mazenc and M. Malisoff. Stabilization and robustness analysis for time-varying systems with time-varying delays using a sequential subpredictors approach. Automatica, 82:118-127, 2017.

[13] F. Mazenc and M. Malisoff. Stabilization of nonlinear time-varying systems through a new prediction based approach. IEEE Transactions on Automatic Control, 62(6):2908-2915, 2017.

[14] F. Mazenc and M. Malisoff. Continuous-discrete sequential observers under sampling and input delays. In Proceedings of the American Control Conference, pages 5156-5160, Milwaukee, WI, 2018.

[15] F. Mazenc, M. Malisoff, and Z. Lin. Further results on input-to-state stability for nonlinear systems with delayed feedbacks. Automatica, 44(9):2415-2421, 2008.

[16] M. Merad, R. Downey, S. Obuz, and W. Dixon. Isometric torque control for neuromuscular electrical stimulation with time-varying input delay. IEEE Transactions on Control Systems Technology, 24(3):971-978, 2016.

[17] W. Michiels and S-I. Niculescu. Stability and Stabilization of TimeDelay Systems. SIAM, Philadelphia, PA, 2007.

[18] P. Pepe. On stability preservation under sampling and approximation of feedbacks for retarded systems. SIAM Journal on Control and Optimization, 54(4):1895-1918, 2016.

[19] P. Pepe. Stabilization of retarded systems of neutral type by control Lyapunov-Krasovskii functionals. Systems and Control Letters, 94(2):142-151, 2016.

[20] J. Peuteman and D. Aeyels. Exponential stability of nonlinear timevarying differential equations and partial averaging. Mathematics of Control, Signals, and Systems, 15:42-70, 2002.

[21] J-P. Richard. Time-delay systems: an overview of some recent advances and open problems. Automatica, 39(10):1667-1694, 2003.

[22] O. Smith. A controller to overcome dead time. ISA-Journal, 6(2):2833, 1958.

[23] J. Weston and M. Malisoff. Sequential predictors under time-varying feedback and measurement delays and sampling. 2018, submitted, https://www.math.lsu.edu/ malisoff/.

[24] B. Zhou. Truncated Predictor Feedback for Time-Delay Systems. Springer-Verlag, Berlin, Germany, 2014.

[25] B. Zhou, Z. Lin, and G-R. Duan. Truncated predictor feedback for linear systems with long time-varying input delays. Automatica, 48:2387-2399, 2012.

[26] B. Zhou, Q. Liu, and F. Mazenc. Stabilization of linear systems with both input and state delays by observer-predictors. Automatica, 83:368-377, 2017. 